\title{
Quantification of Saquinavir
} from Lysates of Peripheral Blood Mononuclear Cells Using Microarrays and Standard MALDITOF-MS

\section{Journal Article}

Author(s):

Pabst, Martin; Fagerer, Stephan R.; Köhling, Rudolf; Eyer, Klaus (iD; Krismer, Jasmin; Jefimovs, Konstantins; Ibáñez, Alfredo J.; Zenobi, Renato (i)

Publication date:

2014-06-01

Permanent link:

https://doi.org/10.3929/ethz-b-000084455

Rights / license:

In Copyright - Non-Commercial Use Permitted

Originally published in:

Journal of the American Society for Mass Spectrometry 25(6), https://doi.org/10.1007/s13361-014-0875-2 


\title{
Quantification of Saquinavir from Lysates of Peripheral Blood Mononuclear Cells Using Microarrays and Standard MALDI-TOF-MS
}

\author{
Martin Pabst, ${ }^{1}$ Stephan Rupert Fagerer, ${ }^{1}$ Rudolf Köhling, ${ }^{2}$ Klaus Eyer, ${ }^{1}$ Jasmin Krismer, ${ }^{1}$ \\ Konstantins Jefimovs, ${ }^{3}$ Alfredo Jesus Ibáñez, ${ }^{1}$ Renato Zenobi ${ }^{1}$
}

${ }^{1}$ Department of Chemistry and Applied Biosciences, ETH Zürich, 8093 Zürich, Switzerland

${ }^{2}$ Sigma-Aldrich, 9470 Buchs, Switzerland

${ }^{3}$ Swiss Federal Laboratories for Material Science and Technology EMPA, 8600 Dübendorf, Switzerland

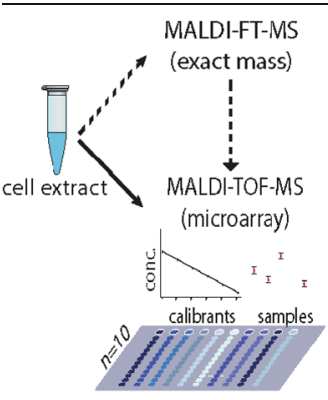

Abstract. Drug monitoring is usually performed by liquid chromatography coupled with optical detection or electrospray ionization mass spectrometry. More recently, matrixassisted laser desorption/ionization (MALDI) in combination with triple quadrupole or Fourier-transform (FT) mass analyzers has also been reported to allow accurate quantification. Here, we present a strategy that employs standard MALDI time-of-flight (TOF) mass spectrometry (MS) for the sensitive and accurate quantification of saquinavir from an extract of blood peripheral mononuclear cells. Unambiguous identification of saquinavir in the mass spectra was possible because of using internal mass calibration and by an overall low chemical noise in the low mass range. Exact mass determination of the constant background peaks of the cell extract, which were used for recalibration, was performed by an initial MALDI-FT-MS analysis. Fast and multiplexed sample analysis was enabled by microarray technology, which provided 10 replicates in the lower $\mathrm{nL}$ range for each sample in parallel lanes on a chip. In order to validate the method, we employed various statistical tests, such as confidence intervals for linear regressions, three quality control samples, and inverse confidence limits of the estimated concentration ratios. Keywords: Quantitative MALDI-MS, Saquinavir, Cell lysate analysis, Peripheral blood mononuclear cells, Microarrays for mass spectrometry (MAMS), Antiretroviral drugs

Received: 2 November 2013/Revised: 19 February 2014/Accepted: 22 February 2014/Published online: 8 April 2014

\section{Introduction}

$\mathrm{T}^{\mathrm{s}}$ he limiting factors in the analysis of antiretroviral drugs are the minute amounts of blood peripheral mononuclear cells (PBMCs) from patients that are available, as well as the complex sample matrix from cell lysates. Therefore, highly sensitive as well as selective detection methods are required. "Gold standards" rely on "high performance" liquid chromatography (HPLC) in combination with UV detection or electrospray mass spectrometry (ESI-MS) [1-3]. However, sample throughput is limited because of the long cycle times of chromatographic runs. By contrast, using MALDI-MS, the analysis of one sample (i.e., one spot) merely takes a few seconds and, therefore, highthroughput analysis is possible. MALDI-MS also is less

Electronic supplementary material The online version of this article (doi:10.1007/s13361-014-0875-2) contains supplementary material, which is available to authorized users.

Correspondence to: Renato Zenobi; e-mail: zenobi@org.chem.ethz.ch demanding with respect to sample preparation or purification and allows preferred ionization of selected classes of compounds depending on the used MALDI matrix. On the other hand, MALDI-MS is usually not the method of choice for quantitative applications [4-6]. Nevertheless, in recent years improvements in sample preparation by introducing modified sample targets or protocols, have efficiently addressed this problem. Furthermore, MALDI instrumentation using triple quadrupole and FT-MS analyzers is increasingly used for quantitative applications and has also been mentioned in clinical drug monitoring [7-15].

Here, we report on a method to quantify saquinavir from a cell extract using standard MALDI-TOF-MS. We performed an initial high resolution mass scan of the cell background using a MALDIFT-MS. Sample analysis was carried out by MALDI-TOF-MS, where spectra were recalibrated using background peaks from reserpine and the cell extract. The overall noise level (e.g., obtained by matrix clusters or fragments) was found to be quite low, although $\alpha$-cyano-4-hydroxycinnamic acid (CHCA) was used as the MALDI matrix (Supplementary Information Figure 1). 
Importantly, the method presented relies on the assistance of microarray plates, which feature multiple lanes of small hydrophilic spots. The plates support the analysis of a large number of replicates, which in turn increases confidence. To evaluate the performance of our method, we plotted the $95 \%$ confidence interval for the calibration series above and below the curve fitted by linear regression. The correlation coefficient $\left(\mathrm{r}^{2}\right)$ was evaluated by plotting all data points measured for the calibration, instead of using average values.

\section{Experimental}

\section{Chemicals}

Alpha-cynao-4-hydroxycinnamic acid (CHCA), saquinavir, atazanavir, indinavir, nelfinavir, and reserpine were purchased from Sigma Aldrich (Buchs, Switzerland).

\section{Microarrays and Sliding Device}

Microarray targets (MAMS, Microarrays for Mass Spectrometry, Supplementary Information Figure 2) were produced as previously described. In brief, the targets were made of ITO-coated microscope glass slides with an omniphobic polysilazane coating on top. Lanes of microarray spots with a diameter of 400 or $500 \mu \mathrm{m}$ and a cavity of $35 \mu \mathrm{m}$ were prepared by picosecond laser ablation $[4,16]$. ITO slides were mounted onto a standard $A B$ SCIEX sample target carrier using a metal mask that was prepared in-house. The sliding device was produced in-house and is made of stainless steel. It consists of an approximately $10 \mathrm{~cm}$ long edge and supporting bars on a guiding rail. The edge is positioned sufficiently low so as to contact the series of droplets that are located in the deposition spots. Then, the slider drags the droplets from the deposition spot over the lanes. Each time the droplet passes over a hydrophilic spot, a nanoliter aliquot is retained, thus generating multiple replicates from one droplet. In total, many replicates can be produced by one sliding motion (the microarray target used herein generated 12 replicates per sample).

\section{Cell Extract}

PBMCs were purchased from ZenBio and lysed as described by van Kampen et al. [11]. In brief, 1 million cells were lysed in $100 \mu \mathrm{L}$ of $50 \%$ ethanol. Lysis was performed without agitation over night at $5^{\circ} \mathrm{C}$. Cells were centrifuged at $14,000 \mathrm{~g}$ at $5^{\circ} \mathrm{C}$ for $10 \mathrm{~min}$, and the supernatant was collected and brought to $20 \%$ $\mathrm{EtOH}$ in $\mathrm{H}_{2} \mathrm{O}$. The cell lysate was further purified using OasisHLB (Waters, London, UK) extraction columns. The eluate from the extraction columns was dried using a speed-vac, redissolved in $10 \mu \mathrm{L}$ spike solution ( $50 \%$ acetonitrile in $\mathrm{H}_{2} \mathrm{O}$ ), internal standard was added, and the samples were mixed with MALDI matrix solution at a ratio of 1 to $2(\mathrm{v} / \mathrm{v})$.

\section{Sample Aliqouting}

A mixture of $4.5 \mu \mathrm{L}$ was pipetted onto the "deposition reservoir" of each lane and aliquoted using a metal slider as described in the materials and methods section as well as in Pabst et al. 2013 [4]. CHCA was used as a MALDI matrix at a concentration of $4 \mathrm{mg}$ per $\mathrm{mL}$ in $60 \%$ acetonitrile and $40 \% \mathrm{H}_{2} \mathrm{O}$, (v/v) with $0.1 \%$ TFA. The slider and microarray target were cooled to $4{ }^{\circ} \mathrm{C}$ for at least $15 \mathrm{~min}$ before aliquoting. This was found to be beneficial to prevent the samples of the nanoliter volumes from drying too fast. Aliquoting was carried out at the laboratory bench, maintaining a constant speed of the slider.

\section{MALDI Mass Spectrometry}

MALDI mass spectra were recorded on a commercial instrument (AB SCIEX TOF/TOFTM 5800 System, Darmstadt, Germany). For MALDI-MS analysis, the whole spot was scanned with a circular scan pattern of 120 individual positions and 20 shots per position (2400 shots in total per spot) at a repetition rate of $1000 \mathrm{~Hz}$, detected in positive reflectron mode. Laser energy was set to 3800 (arbitrary units, as defined by the AB SCIEX software). Spectra were averaged and relative signal areas calculated by dividing the signal area of saquinavir by that of reserpine. The concentration ratios were then used for generating the calibration curve. Internal mass calibration of the MALDI-MS instrument was carried out using peaks from reserpine and constant background signals. Exact masses were obtained by measuring one reference sample with internal calibration on a Bruker SolariX, as described in Steinhoff et al. [17]. All 12 replicates of each data point were measured. The first and last spots of each row were used for performing MS/MS experiments and for tuning the MALDI-TOF-MS instrument.

\section{Internal Standards and Generation of Calibration Curves}

As an internal standard for saquinavir, we used reserpine; for atazanavir and nelfinavir, we used indinavir, and for indinavir, nelfinavir was chosen. Five-point calibration curves of standard solutions (in 50\% acetonitrile) were acquired for all antiretroviral drugs between concentrations of 15.6 and $2000 \mathrm{ng}$ per $\mathrm{mL}$.

Saquinavir was also quantified from an extract of blood peripheral mononuclear cells, using reserpine as an internal standard. The estimation of the concentration ratios of the quality control samples for saquinavir was carried out using an external calibration method. Ten $\mu \mathrm{L}$ of standard solutions with concentrations of $15.6,125,500,1500,3000$, and $4000 \mathrm{ng} / \mathrm{mL}$ with a constant concentration of $750 \mathrm{ng} / \mathrm{mL}$ reserpine were spiked to a cell extract of $1 \times 10^{6}$ cells. If we assume a volume of $0.4 \mathrm{pL}$ per cell [18], these concentrations would translate into PBMC concentrations of $0.39,3.125,12.5,37.5,75$, and $100 \mathrm{ng} / \mathrm{mL}$. As estimated in Pabst et al. 2013 [4], each array spot contains approximately $10 \mathrm{~nL}$. Therefore, the lowest concentration (15.6 ng per $\mathrm{mL}$ ) equals an absolute amount of approximately 250 attomol saquinavir per array spot.

\section{Data Analysis}

Data extraction from MALDI-TOF analysis results was carried out using the analysis tool of the TOF/TOFseries Explorer 
Table 1. Relative signal intensities of saquinavir for three quality control samples

\begin{tabular}{|c|c|c|c|c|c|c|c|}
\hline $\begin{array}{l}\mathrm{A}_{\text {rel }} \\
\left(\mathrm{A}_{\text {Saq }} / \mathrm{A}_{\text {Res }}\right)\end{array}$ & $\begin{array}{l}\text { Estimated conc. } \\
\mathrm{ng} / \mathrm{mL}\end{array}$ & $\begin{array}{l}\text { St. dev. (RSD \%) } \\
(n=10)\end{array}$ & $\begin{array}{l}\text { Nominal conc. } \\
\mathrm{ng} / \mathrm{mL}\end{array}$ & $\begin{array}{l}\text { Accuracy } \\
\% \text { error }\end{array}$ & $\begin{array}{l}95 \% \text { Inverse } \\
\text { confidence limits }\end{array}$ & $\begin{array}{l}\text { Mass accuracy (ppm) } \\
\text { saquinavir } \\
{[\mathrm{M}+\mathrm{H}]^{+}=671.4}\end{array}$ & $\begin{array}{l}\text { Signal-to-noise ratio } \\
\text { saquinavir } \\
{[\mathrm{M}+\mathrm{H}]^{+}=671.4}\end{array}$ \\
\hline 2.010 & 53.28 & $2.4(4.54 \%)$ & 50.0 & 6.5 & 0.97 & 2.57 & 3166 \\
\hline 1.027 & 27.25 & $0.84(3.1 \%)$ & 25.0 & 9.0 & 0.97 & 2.97 & 2094 \\
\hline 0.046 & 1.29 & $0.04(3.29 \%)$ & 1.5 & 17.1 & 1.03 & 9.8 & 485 \\
\hline
\end{tabular}

Estimated concentrations were within $6.5 \%$ and $17.1 \%$ relative error of the nominal values. Standard deviations of the repeated measurements are relatively low and confidence limits of the estimated sample concentration appear narrow. Mass accuracy was found within 10 ppm even for the lowest concentration

software from AB SCIEX. Calculation of the $95 \%$ confidence interval, $\mathrm{r}^{2}$, curve of the linear regression, and inverse confidence limits for quality control samples were all done using Microsoft Excel. All points (i.e., the whole data cloud) were plotted for data analysis as opposed to averaging all points for a given concentration. The formulae for inverse confidence limits were taken from Szajli et al. [19] and Eisenhart et al. [20]. Outlier tests were performed using a Q-test, for which up to one data point was allowed to be treated as an outlier per 10 data points.

\section{Results and Discussion}

In a first set of experiments, we evaluated the sensitivity and usefulness of our system for the analysis of selected antiretroviral drugs (saquinavir, atazanavir, indinavir, and nelfinavir) using CHCA (Supplementary Information Figure 3). By using the microarray, we obtained quite low levels of chemical noise in the low $\mathrm{m} / \mathrm{z}$ range, which was vital for the unambiguous assignment of analyte signals that are close to the limit of quantification (Fig. 1, Supplementary Information Figure 1). We were able to obtain calibration curves with low standard deviations and $\mathrm{r}^{2}$ values greater than 0.98 for all antiretroviral drugs (Supplementary Information Figure 3).

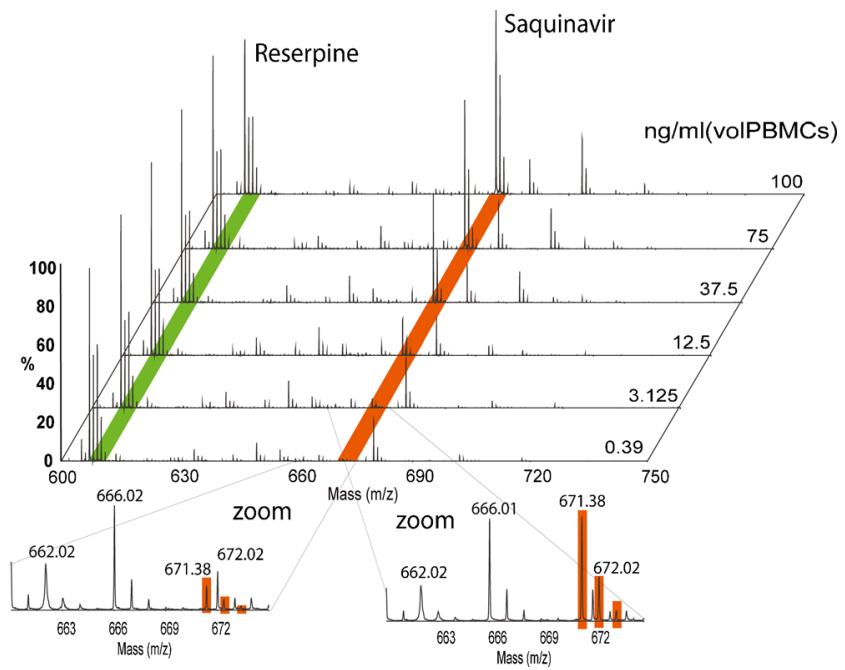

Figure 1. Mass spectra series from $0.39 \mathrm{ng} / \mathrm{mL}$ and $100 \mathrm{ng} / \mathrm{mL}$ (volume PBMCs) plotted. A zoom of the two lowest concentrations is presented below. Saquinavir is highlighted with orange bars. Reserpine, which was used as internal standard, is highlighted with a green bar
We finally evaluated the practicability of employing standard MALDI-TOF-MS for quantifying saquinavir in the complex matrix of a cell extract. In a single pre-scan, we determined the accurate masses of the constant background peaks by MALDI-FT-MS once and chose four peaks close to the mass of saquinavir $\left[(\mathrm{M}+\mathrm{H})^{+}=679.1\right]$ for further internal calibration of the MALDI-TOF-MS spectra (Supplementary Information Figure 4). Therefore, it was not necessary to add other internal standards, which might have resulted in a loss of sensitivity because of competitive ionization. Saquinavir was identified within a $10 \mathrm{ppm}$ mass window, even at the lowest concentration (Supplementary Information Table 1). Mass resolution for the MALDI-TOF-MS instrument was found to be about 10,000 (FWHM). Moreover, the identity of the analytes was confirmed by additional MS/MS experiments.

Average standard deviations, mass accuracy, and signal-tonoise ratios for the data points of the calibration curve can be found in Supplementary Information Table 1). Alongside the calibration curve, we analyzed three quality-control samples for saquinavir. For this, we obtained relative standard deviations for the replicate measurements of $4.5 \%, 3.1 \%$, and $3.3 \%$ for the lowest concentration $(1.5 \mathrm{ng} / \mathrm{mL})$. The accuracy for the independent quality control samples were further found to be

\section{Regression using all data points}

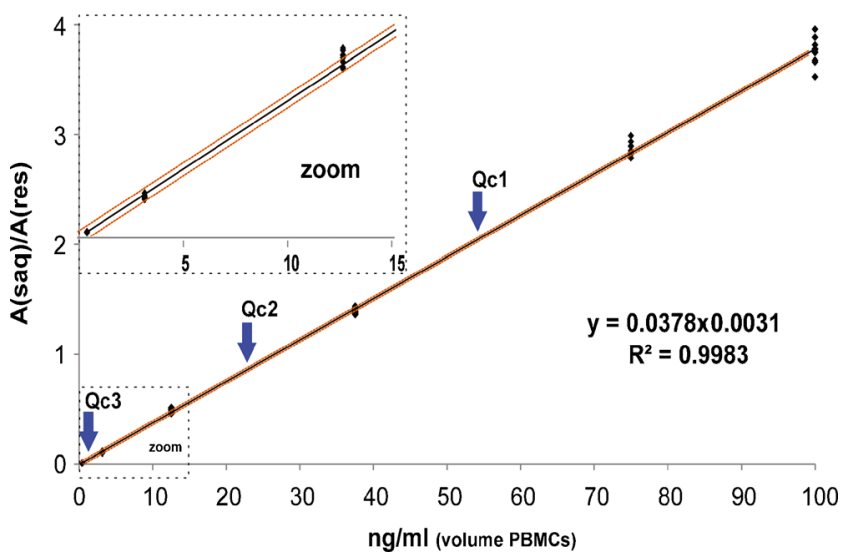

Figure 2. Calibration curve obtained after plotting the whole data series for saquinavir spiked to extracts of PBMCs $(n=10$ per data point). The series was linear between $0.39 \mathrm{ng} / \mathrm{mL}$ and $100 \mathrm{ng} / \mathrm{mL}$ (volume of PBMCs expressed in $\mathrm{mL}$ ); $95 \%$ confidence intervals for the curve of best fit are outlined as orange lines. The $r^{2}$ value was calculated considering the whole data series in the plot $(n=60)$ in graph. Points for quality control samples (1-3) are marked with blue arrows 
6.5\% (53.28 $\pm 2.4 \mathrm{ng} / \mathrm{mL}), 9 \%(27.25 \pm 0.84 \mathrm{ng} / \mathrm{mL})$, and $17 \%$ $(1.29 \pm 0.04 \mathrm{ng} / \mathrm{mL})$ (Table 1 and Supplementary Information Figure 5). As expected for a confident analysis, the confidence intervals of the curve of best fit and calculated $95 \%$ inverse confidence limits according, to Szajli et al. [19] and Eisenhart et al. [20], appeared very narrow (Fig. 2, Supplementary Information Figure 6). We attribute the high data quality to the large number of replicates ( $n=60$ for the calibration curve, plus 10 for the quality control sample), and the overall low standard deviations of the MALDI-MS analysis (on average 3.9\%, Supplementary Information Table 1). In the theoretical case of using a much lower number of replicates (e.g., $n=18$ for the calibration curve if three replicates per data point are acquired), both confidence limits would increase drastically, even if standard deviations and the correlation of the regression curve might be acceptable. Confidence limits, therefore, allow a reliable decision to be made on the quality of the results of the analysis [19]. The whole chip was aliquoted within a few seconds (six calibration points and three quality control samples). Furthermore, we were able to acquire 10 replicates in approximately $1.5 \mathrm{~min}$, which is much faster than what can be reached by methods involving chromatographic separations.

\section{Conclusions}

By combining a suitable sample preparation with microarray technology and internal mass calibration, we demonstrated that accurate, precise, and confident quantification of antiretroviral drugs can be achieved by using standard MALDI-TOF-MS. A single pre-scan of the accurate masses as well as a low background signal noise in the lower mass range allowed ambiguous peak assignment and quantification from a complex matrix (Figs. 1 and 2).

\section{Acknowledgments}

This project was co-financed by the Swiss KTI (Kommission für Technologie und Innovation), grant no. 13123.1 PFNM-NM. The authors additionally thank Robert Steinhoff and Simon Weidmann for constructive discussions as well as helpful advice during our MALDI-MS measurements and Rolf Häflinger for determining exact masses from the reference samples.

M.P., S.F., R.K., K.J., and R.Z. are developing a commercial product based on the microarray for mass spectrometry technology.

\section{References}

1. Jung, B.H., Rezk, N.L., Bridges, A.S., Corbett, A.H., Kashuba, A.D.: Simultaneous determination of 17 antiretroviral drugs in human plasma for quantitative analysis with liquid chromatography-tandem mass spectrometry. Biomed. Chromatogr. 21(10), 1095-1104 (2007)

2. Koal, T., Burhenne, H., Romling, R., Svoboda, M., Resch, K., Kaever, V.: Quantification of antiretroviral drugs in dried blood spot samples by means of liquid chromatography/tandem mass spectrometry. Rapid Commun. Mass Spetrom. 19(21), 2995-3001 (2005)

3. Sarkar, M., Khandavilli, S., Panchagnula, R.: Development and validation of rp-hplc and ultraviolet spectrophotometric methods of analysis for the quantitative estimation of antiretroviral drugs in pharmaceutical dosage forms. J. Chromatogr. B 830(2), 349-354 (2006)

4. Pabst, M., Fagerer, S.R., Kohling, R., Kuster, S.K., Steinhoff, R., Badertscher, M., Wahl, F., Dittrich, P.S., Jefimovs, K., Zenobi, R.: Selfaliquoting microarray plates for accurate quantitative matrix-assisted laser desorption/ionization mass spectrometry. Anal. Chem. 85(20), 9771-9776 (2013)

5. Sleno, L., Volmer, D.A.: Assessing the properties of internal standards for quantitative matrix-assisted laser desorption/ionization mass spectrometry of small molecules. Rapid Commun. Mass Spectrom. 20(10), $1517-1524$ (2006)

6. Wu, J., Chatman, K., Harris, K., Siuzdak, G.: An automated MALDI mass spectrometry approach for optimizing cyclosporin extraction and quantitation. Anal. Chem. 69(18), 3767-3771 (1997)

7. Meesters, R.J., van Kampen, J.J., Reedijk, M.L., Scheuer, R.D., Dekker, L.J., Burger, D.M., Hartwig, N.G., Osterhaus, A.D., Luider, T.M., Gruters, R.A.: Ultrafast and high-throughput mass spectrometric assay for therapeutic drug monitoring of antiretroviral drugs in pediatric hiv-1 infection applying dried blood spots. Anal. Bioanal. Chem. 398(1), 319-328 (2010)

8. Meesters, R.J., van Kampen, J.J., Scheuer, R.D., van der Ende, M.E., Gruters, R.A., Luider, T.M.: Determination of the antiretroviral drug tenofovir in plasma from hiv-infected adults by ultrafast isotope dilution maldi-triple quadrupole tandem mass spectrometry. J. Mass Spectrom. 46(3), 282-289 (2011)

9. Notari, S., Mancone, C., Alonzi, T., Tripodi, M., Narciso, P., Ascenzi, P.: Determination of abacavir, amprenavir, didanosine, efavirenz, nevirapine, and stavudine concentration in human plasma by MALDITOF/TOF. J. Chromatogr. B 863(2), 249-257 (2008)

10. Notari, S., Mancone, C., Tripodi, M., Narciso, P., Fasano, M., Ascenzi, P.: Determination of anti-HIV drug concentration in human plasma by MALDI-TOF/TOF. J. Chromatogr. B 833(1), 109-116 (2006)

11. van Kampen, J.J., Burgers, P.C., de Groot, R., Luider, T.M.: Qualitative and quantitative analysis of pharmaceutical compounds by MALDITOF mass spectrometry. Anal. Chem. 78(15), 5403-5411 (2006)

12. van Kampen, J.J., Burgers, P.C., de Groot, R., Osterhaus, A.D., Reedijk, M.L., Verschuren, E.J., Gruters, R.A., Luider, T.M.: Quantitative analysis of HIV-1 protease inhibitors in cell lysates using maldifticr mass spectrometry. Anal. Chem. 80(10), 3751-3756 (2008)

13. van Kampen, J.J., Reedijk, M.L., Burgers, P.C., Dekker, L.J., Hartwig, N.G., van der Ende, I.E., de Groot, R., Osterhaus, A.D., Burger, D.M., Luider, T.M., Gruters, R.A.: Ultra-fast analysis of plasma and intracellular levels of hiv protease inhibitors in children: a clinical application of maldi mass spectrometry. PloS One 5(7), e11409 (2010)

14. Wagner, M., Varesio, E., Hopfgartner, G.: Ultra-fast quantitation of saquinavir in human plasma by matrix-assisted laser desorption/ ionization and selected reaction monitoring mode detection. J. Chromatogr. B 872(1/2), 68-76 (2008)

15. van Kampen, J.J., Burgers, P.C., Gruters, R.A., Osterhaus, A.D., de Groot, R., Luider, T.M., Volmer, D.A.: Quantitative analysis of antiretroviral drugs in lysates of peripheral blood mononuclear cells using maldi-triple quadrupole mass spectrometry. Anal. Chem. 80(13), 4969-4975 (2008)

16. Urban, P.L., Jefimovs, K., Amantonico, A., Fagerer, S.R., Schmid, T., Madler, S., Puigmarti-Luis, J., Goedecke, N., Zenobi, R.: High-density micro-arrays for mass spectrometry. Lab Chip 10(23), 3206-3209 (2010)

17. Steinhoff, R.F., Krismer, J., Eyer, K., Fagerer, S.R., Ibanez, A., Pabst, M., Zenobi, R.: Rapid estimation of the energy charge from cell lysates using matrix-assisted laser desorption/ionization mass spectrometry: role of in-source fragmentation. Anal. Biochem. 447, 107-113 (2014)

18. Colombo, S., Beguin, A., Telenti, A., Biollaz, J., Buclin, T., Rochat, B., Decosterd, L.A.: Intracellular measurements of anti-hiv drugs indinavir, amprenavir, saquinavir, ritonavir, nelfinavir, lopinavir, atazanavir, efavirenz, and nevirapine in peripheral blood mononuclear cells by liquid chromatography coupled to tandem mass spectrometry. J. Chromatogr. B 819(2), 259-276 (2005)

19. Szajli, E., Feher, T., Medzihradszky, K.F.: Investigating the quantitative nature of maldi-tof ms. Mol. Cell. Proteom. 7(12), 2410-2418 (2008)

20. Eisenhart, C.: The interpretation of certain regression methods and their use in biological and industrial research. Ann. Math. Stat. 10(2), 162-186 (1939) 